\title{
Accuracy Limits and Mobile Terminal Selection Scheme for Cooperative Localization in Cellular Networks
}

\author{
Ziming He, Yi Ma, and Rahim Tafazolli \\ Centre for Communication Systems Research \\ University of Surrey, Guildford, UK \\ e-mail:\{z.he, y.ma, r.tafazolli\}@surrey.ac.uk
}

\begin{abstract}
This paper consider cooperative localization in cellular networks. In this scenario, several located mobile terminals (MTs) are employed as reference nodes to find the location of an un-located MT. The located MTs sent training sequences in the uplink, then the un-located MT perform distance estimation using received signal strength techniques. The localization accuracy of the un-located MT is characterized in terms of squared position error bound (SPEB) [1]. By taking into account the imperfect a priori location knowledge of the located MTs, the SPEB is derived in a closed-form. The closed-form indicate that the effect of the imperfect location knowledge on SPEB is equivalent to the increase of the variance of distance estimation. Moreover, based on the obtained closed-form, a MT selection scheme is proposed to decrease the number of located MTs sending training sequences, thus reduce the training overhead for localization. The simulation results show that the proposed scheme can reduce the training overhead with the paid of accuracy. And with the same training overhead, the accuracy of the proposed scheme is better than that of random selection.
\end{abstract}

\section{INTRODUCTION}

Cellular network based localization techniques have been extensively investigated over the past decade, e.g., [2]-[7]. Previous approaches includes time-of-arrival (TOA) [2], timedifference-of-arrival (TDOA) [3] and received signal strength (RSS) techniques [4]. The major difference between TOA and TDOA approaches is: TOA approaches require tight clock synchronization between base-station (BS) and mobile terminal (MT), while TDOA approaches only require tight clock synchronization among BSs. Many of the wireless standards only mandate timing clock synchronization among BSs, the MT clock might have a drift of a few microseconds [8]. One of the advantages of RSS approach is that it does not require clock synchronization.

The training signals for location estimation can be sent either by BS through forward link (i.e downlink) or MT through reverse link (i.e uplink). Take TDOA-based approach as an example, most state-of-the-arts focus on downlink TDOA, such as Enhanced-Observed Time Difference (E-OTD) for Global System for Mobile Communications (GSM), Observed TDOA (OTDOA) for Universal Mobile Telecommunications System (UMTS) [3]. Since Orthogonal frequency-division multiplexing (OFDM) is a candidate for future mobile networks, downlink TDOA is investigated in OFDM cellular network in [5]. Another alternative is uplink TDOA (U-TDOA) approaches [6], which employs location measurement unit (LMU) at BSs to listen to the training signals sent by the MTs in uplink.

Cooperative localization has been extensively investigated in wireless sensor networks (WSN) [9]-[10]. Recently, the concept of cooperative localization is introduced to cellular networks [11]-[13]. The work in [11] and [13] assume that clock synchronization is perfect between MTs. Then the distances between MTs are estimated using TOA techniques. A more practical cooperative localization approach for cellular network is proposed in [12]. Their work employs located MTs to serve as reference nodes. The located MTs sent training sequences to an un-located MT for location estimation. However, their work did not investigate localization accuracy of this approach. Since each located MT sents training sequences for distance estimation, the number of training sequences increases with the increases of the located MTs. The number of training sequences for localization is overhead for communications. Therefore, the overhead should be reduced, otherwise the efficiency of communication is decreased.

This paper consider a single cell scenario as depicted in Fig. 1. The home BS is responsible for collecting the distance estimates and perform location estimation. There are several located MTs, whose locations are known a priori at the home BS, and there is an un-located MT without any location knowledge. The a priori location knowledge comes from successive localization or tracking [1], and they are usually imperfect. The located MTs sents training sequences for the un-located MT to perform distance estimation using RSS techniques. Then, the home BS collects the distance estimates and calculate the location of the un-located MT.

The major contributions of this paper are: 1) The localization accuracy of the un-located MT is characterized in terms of squared position error bound (SPEB) [1]. By taking into account the imperfect a priori location knowledge of the located MTs, the SPEB is derived in a closed-form. The closed-form indicate that, the effect of the imperfect knowledge on SPEB is equivalent to the increase of the variance of RSS-based distance estimation. 2) A MT selection scheme is proposed using the obtained closed-form. Utilizing the a priori location 


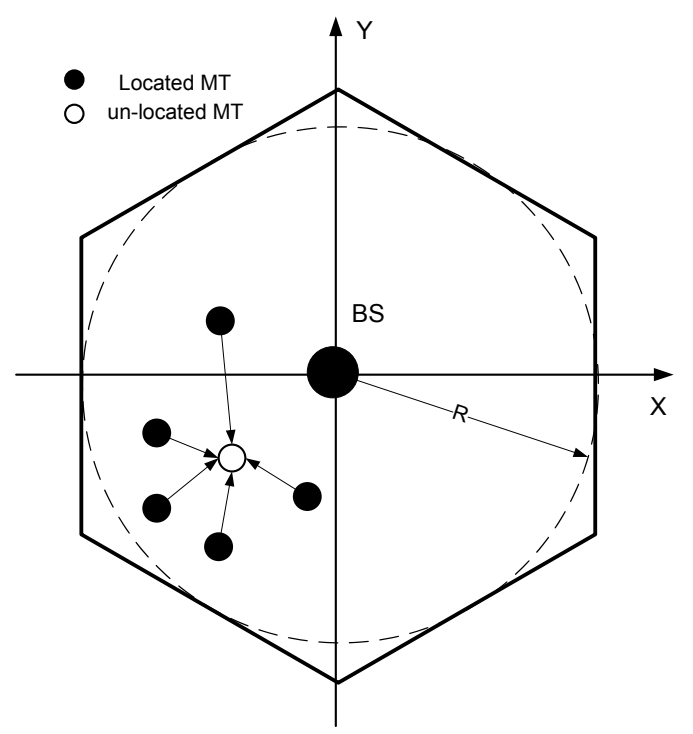

Fig. 1. Scenario and System Model

knowledge, the proposed scheme allow the home BS to select some of the located MTs to send training sequences. Thus, the training overhead is reduced. The simulation results show that the proposed scheme can reduce the training overhead with the paid of accuracy. And with the same training overhead, the accuracy of the proposed scheme is better than that of random selection.

\section{System Description AND SPEB}

\section{A. System Description}

There are $N$ located MTs sending training sequences for location estimation. Each of the MTs sends only one training sequence for a single location estimation, and the training sequences sent by different located MTs are orthogonal either in time or frequency to avoid collision.

Denote $\theta_{n} \triangleq\left(x_{n}, y_{n}\right)$ as the 2-D true location of the $n$-th located MTs $(n \in[1, N]), \theta \triangleq(x, y)$ as the true location of the un-located MT, $p\left(\theta_{1}, \ldots, \theta_{N}\right)$ as the joint p.d.f of the a priori locations knowledge of located MTs, where $x$ and $x_{n}$ denote X coordinates, and $y$ and $y_{n}$ denote $\mathrm{Y}$ coordinates. $p\left(\theta_{1}, \ldots, \theta_{N}\right)$ is known at the home BS. The considered problem can be formulated as $N+1$ nodes cooperative localization with $a$ priori location knowledge. The parameters of interests is given by $\boldsymbol{\theta}=\left[\theta_{1}, \ldots, \theta_{N}, \theta\right]^{T}$.

Denotes $\mathbf{P}_{\mathbf{r}}, \mathbf{P}_{\mathbf{t}}, \mathbf{L}$ as $N \times 1$ vectors collect the RSS at the un-located MT, transmitted power of the located MTs, and path loss in $\mathrm{dB}$ scale, respectively. $N \times 1$ vectors $\mathbf{e}$ are independent Gaussian random variable representing lognormal fading. Denote $\sigma$ as the standard deviation of the element in e. In a typical case, $\sigma=6-8$ (dB) [14]. Then, the following equation holds [15]

$$
\mathbf{P}_{\mathbf{r}}=\mathbf{P}_{\mathbf{t}}-\mathbf{L}+\mathbf{e}
$$

where

$$
\mathbf{L}=10 \gamma \log _{10} \mathbf{d}
$$

$$
d_{n}=\sqrt{\left(x_{n}-x\right)^{2}+\left(y_{n}-y\right)^{2}}
$$

$d_{n}$ denotes the $n$-th element in $\mathbf{d}$.

\section{B. CRLB Formulation}

In order to derive SPEB, we will first formulate the CramérRao lower bound (CRLB). The CRLB with a priori knowledge reads [16, Page 84]

$$
\mathbb{E}_{\mathbf{P}_{\mathbf{r}}, \boldsymbol{\theta}}\left[(\hat{\boldsymbol{\theta}}-\boldsymbol{\theta})(\hat{\boldsymbol{\theta}}-\boldsymbol{\theta})^{T}\right] \geq \mathbf{J}^{-1},
$$

where

$$
\mathbf{J}=\mathbf{J}_{\boldsymbol{\theta}}+\mathbf{J}_{\mathbf{P}}
$$

$\hat{\boldsymbol{\theta}}$ denotes estimates of $\boldsymbol{\theta}, \mathbb{E}_{\mathbf{P}_{\mathbf{r}}, \boldsymbol{\theta}}[\cdot]$ the expectation with respect to $\mathbf{P}_{\mathbf{r}}$ and $\boldsymbol{\theta}, \mathbf{J}$ the Fisher information matrix (FIM), $\mathbf{J}_{\boldsymbol{\theta}}$ the FIM from the observations [1] and has the following expression

$$
\mathbf{J}_{\boldsymbol{\theta}}=\mathbb{E}_{\hat{\mathbf{d}}}\left[\frac{\partial}{\partial \boldsymbol{\theta}} \ln p\left(\mathbf{P}_{\mathbf{r}} \mid \boldsymbol{\theta}\right)\left(\frac{\partial}{\partial \boldsymbol{\theta}} \ln p\left(\mathbf{P}_{\mathbf{r}} \mid \boldsymbol{\theta}\right)\right)^{T}\right],
$$

$\mathbf{J}_{\mathbf{P}}$ the FIM from the a priori knowledge [1] and has the following expression

$$
\mathbf{J}_{\mathbf{P}}=\mathbb{E}_{\boldsymbol{\theta}}\left[\frac{\partial}{\partial \boldsymbol{\theta}} \ln p(\boldsymbol{\theta})\left(\frac{\partial}{\partial \boldsymbol{\theta}} \ln p(\boldsymbol{\theta})\right)^{T}\right],
$$

$p(\boldsymbol{\theta})$ the joint p.d.f of a priori knowledge of $\boldsymbol{\theta}$. Since the un-located MT do not have any a priori location knowledge, $p(\boldsymbol{\theta})=p\left(\theta_{1}, \ldots, \theta_{N}\right)$. According to [17], $\mathbf{J}_{\boldsymbol{\theta}}$ can be written as

$$
\mathbf{J}_{\boldsymbol{\theta}}=\frac{1}{\varepsilon^{2}} \mathbf{H}^{T} \mathbf{H}
$$

where $\mathbf{H}=\nabla_{\boldsymbol{\theta}}^{T} \otimes \mathbf{d}\left(\otimes\right.$ denotes the Kronecker product, $\nabla_{\boldsymbol{\theta}} \triangleq$ $\left.\left[\frac{\partial}{\partial x_{1}}, \frac{\partial}{\partial y_{1}}, \ldots, \frac{\partial}{\partial x_{N}}, \frac{\partial}{\partial y_{N}}, \frac{\partial}{\partial x}, \frac{\partial}{\partial y}\right]^{T}\right), \varepsilon=(\sigma \ln 10) /(10 \gamma), \gamma$ the path loss factor. $\gamma=2$ for free space, $\gamma=4$ is often used to characterize the path loss in urban areas [14]. $\mathbf{H}$ can be further expressed as

$$
\mathbf{H}=\left[\begin{array}{ll}
\mathbf{G} & \mathbf{U}
\end{array}\right]
$$

where the elements of $\mathbf{G}$ and $\mathbf{U}$ are zeros, expect for the following elements

$$
\begin{gathered}
\left\{\begin{array}{l}
\mathbf{G}(n, 2 n-1)=\frac{\cos \phi_{n}}{d_{n}} \\
\mathbf{G}(n, 2 n)=\frac{\sin \phi_{n}}{d_{n}}
\end{array}\right. \\
\left\{\begin{array}{l}
\mathbf{U}(n, 1)=-\frac{\cos \phi_{n}}{d_{n}} \\
\mathbf{U}(n, 2)=-\frac{\sin \phi_{n}}{d_{n}}
\end{array}\right.
\end{gathered}
$$

where $\phi_{n}$ denotes the angle from the $n$-th located MT to the un-located MT, i.e., $\phi_{n}=\tan ^{-1} \frac{y-y_{n}}{x-x_{n}}$. Then, (11) can be further written as

$$
\mathbf{J}_{\boldsymbol{\theta}}=\frac{1}{\varepsilon^{2}}\left[\begin{array}{cc}
\mathbf{G}^{T} \mathbf{G} & \mathbf{G}^{T} \mathbf{U} \\
\mathbf{U}^{T} \mathbf{G} & \mathbf{U}^{T} \mathbf{U}
\end{array}\right]
$$

Assuming $p\left(\theta_{1}, \ldots, \theta_{N}\right)$ is $2 N$-order Gaussian p.d.f, $\mathbf{J}_{\mathbf{P}}$ can be written as [16, Page 85]

$$
\mathbf{J}_{\mathbf{P}}=\left[\begin{array}{cc}
\boldsymbol{\Omega}^{-1} & \mathbf{0} \\
\mathbf{0} & \mathbf{0}
\end{array}\right]
$$


where $\Omega$ denotes the covariance matrix of the $a$ priori location knowledge. For mutually independent a priori knowledge, $\boldsymbol{\Omega}=\operatorname{diag}\left\{\omega_{1}^{2} \mathbf{I}_{2}, \ldots, \omega_{N}^{2} \mathbf{I}_{\mathbf{2}}\right\}$, where $\omega_{n}^{2}$ denotes the variance of a priori knowledge of the $n$-th located MT, $\mathbf{I}_{2} 2 \times 2$ identity matrix. And $\boldsymbol{\omega} \triangleq\left[\omega_{1}^{2}, \ldots, \omega_{N}^{2}\right]$. The FIM can be written as

$$
\begin{aligned}
& \mathbf{J}=\left[\begin{array}{cc}
\frac{1}{\varepsilon^{2}} \mathbf{G}^{T} \mathbf{G}+\boldsymbol{\Omega}^{-1} & \frac{1}{\varepsilon^{2}} \mathbf{G}^{T} \mathbf{U} \\
\frac{1}{\varepsilon^{2}} \mathbf{U}^{T} \mathbf{G} & \frac{1}{\varepsilon^{2}} \mathbf{U}^{T} \mathbf{U}
\end{array}\right] \\
& \triangleq\left[\begin{array}{cc}
\mathbf{A} & \mathbf{B} \\
\mathbf{B}^{T} & \mathbf{C}
\end{array}\right]
\end{aligned}
$$

The CRLB of location estimation of the un-located MT reads

$$
\mathbf{F}=\left[\mathbf{J}^{-1}\right]_{2 \times 2}=(\mathbf{C}-\underbrace{\mathbf{B}^{T} \mathbf{A}^{-1} \mathbf{B}}_{\mathbf{M}})^{-1}
$$

where $\left[\mathbf{J}^{-1}\right]_{2 \times 2}$ denotes the last $2 \times 2$ diagonal submatrix of $\mathbf{J}^{-1}$.

\section{Closed-form of SPEB}

According to [1], the SPEB reads

$$
\mathcal{P}=\operatorname{tr}\{\mathbf{F}\}
$$

If the a priori knowledge is perfect, $\boldsymbol{\omega}=\mathbf{0}$, (16) reduce to

$$
\mathcal{P}=\operatorname{tr}\left\{\mathbf{C}^{-1}\right\}
$$

which is the SPEB for single MT localization with $N$ anchors. In the following, we derive the closed-form of (16) and (17).

Matrix A can be written as

$$
\mathbf{A}=\operatorname{diag}\left\{\mathbf{A}_{\mathbf{1}}, \cdots, \mathbf{A}_{\mathbf{N}}\right\}
$$

where the sub-matrix $\mathbf{A}_{\mathbf{n}}(n \in[1, N])$ is

$$
\mathbf{A}_{\mathbf{n}}=\left[\begin{array}{cc}
\frac{\cos ^{2} \phi_{n}}{\varepsilon^{2} d_{n}^{2}}+\frac{1}{\omega_{n}^{2}} & \frac{\sin \phi_{n} \cos \phi_{n}}{\varepsilon^{2} d_{n}^{2}} \\
\frac{\sin \phi_{n} \cos \phi_{n}}{\varepsilon^{2} d_{n}^{2}} & \frac{\sin ^{2} \phi_{n}}{\varepsilon^{2} d_{n}^{2}}+\frac{1}{\omega_{n}^{2}}
\end{array}\right]
$$

Then the inverse of $\mathbf{A}$ reads

$$
\mathbf{A}^{-1}=\operatorname{diag}\left\{\mathbf{A}_{\mathbf{1}}{ }^{-1}, \cdots, \mathbf{A}_{\mathbf{N}}{ }^{-1}\right\}
$$

where

$$
\mathbf{A}_{\mathbf{n}}{ }^{-1}=\left[\begin{array}{cc}
\frac{\omega_{n}^{4} \sin ^{2} \phi_{n}}{\beta_{n}}+\frac{\varepsilon^{2} \omega_{n}^{2} d_{n}^{2}}{\beta_{n}} & -\frac{\omega_{n}^{4} \sin \phi_{n} \cos \phi_{n}}{\beta_{n}} \\
-\frac{\omega_{n}^{4} \sin \phi_{n} \cos \phi_{n}}{\beta_{n}} & \frac{\cos ^{2} \phi_{n}}{\varepsilon^{2} d_{n}^{2}}+\frac{\varepsilon^{2} \omega_{n}^{2} d_{n}^{2}}{\beta_{n}}
\end{array}\right]
$$

and $\beta_{n}=\omega_{n}^{2}+\varepsilon^{2} d_{n}^{2}$. Since

$$
\mathbf{M}=\frac{1}{\varepsilon^{2}}\left[\begin{array}{cc}
\sum_{n=1}^{N} \frac{\omega_{n}^{2} \cos ^{2} \phi_{n}}{d_{n}^{2} \beta_{n}} & \sum_{n=1}^{N} \frac{\omega_{n}^{2} \sin \phi_{n} \cos \phi_{n}}{d_{n}^{2} \beta_{n}} \\
\sum_{n=1}^{N} \frac{\omega_{n}^{2} \sin \phi_{n} \cos \phi_{n}}{d_{n}^{2} \beta_{n}} & \sum_{n=1}^{N} \frac{\omega_{n}^{2} \sin ^{2} \phi_{n}}{d_{n}^{2} \beta_{n}}
\end{array}\right]
$$

and

$$
\mathbf{C}=\frac{1}{\varepsilon^{2}}\left[\begin{array}{cc}
\sum_{n=1}^{N} \frac{\cos ^{2} \phi_{n}}{d_{n}^{2}} & \sum_{n=1}^{N} \frac{\sin \phi_{n} \cos \phi_{n}}{d^{2}} \\
\sum_{n=1}^{N} \frac{\sin \phi_{n} \cos \phi_{n}}{d_{n}^{2}} & \sum_{n=1}^{N} \frac{\sin ^{2} \phi_{n}}{d_{n}^{2}}
\end{array}\right],
$$

$\mathbf{C}-\mathbf{M}=\left[\begin{array}{cc}\sum_{n=1}^{N} \frac{1}{\beta_{n}} \cos ^{2} \phi_{n} & \sum_{n=1}^{N} \frac{1}{\beta_{n}} \sin \phi_{n} \cos \phi_{n} \\ \sum_{n=1}^{N} \frac{1}{\beta_{n}} \sin \phi_{n} \cos \phi_{n} & \sum_{n=1}^{N} \frac{1}{\beta_{n}} \sin ^{2} \phi_{n}\end{array}\right]$
Finally, the closed-form of (16) reads

$$
\begin{aligned}
& \mathcal{P}= \\
& \frac{\sum_{n=1}^{N} \frac{1}{\beta_{n}}}{\left(\sum_{n=1}^{N} \frac{\cos ^{2} \phi_{n}}{\beta_{n}}\right)\left(\sum_{n=1}^{N} \frac{\sin ^{2} \phi_{n}}{\beta_{n}}\right)-\left(\sum_{n=1}^{N} \frac{\sin \phi_{n} \cos \phi_{n}}{\beta_{n}}\right)^{2}}(25)
\end{aligned}
$$

The closed-form of (17) is simply replacing $\beta_{n}$ in (25) with $\varepsilon^{2} d_{n}^{2}$, which is actually the CRLB of RSS-based distance estimation [18]. Therefore, it is concluded that the effect of the imperfect location knowledge on SPEB is equivalent to the increase of the variance of RSS-based distance estimation.

\section{MT SELECTION SCHEME}

Utilizing the a priori knowledge $\boldsymbol{\omega}$, the home BS select $S$ located MTs out of $N$ located MTs $(S \leq N)$ to sent training sequences. The MT selection scheme is firstly obtained by considering a special case, then the proposed scheme is evaluated for a general case in Section IV. For this special case, $\phi_{n}=2 \pi(n-1) / N, d_{1}=d_{2}=\cdots=d_{n}=d$, the $k$-th located MT is the one the BS does not select, and the other $N-1$ are the selected MTs, and

$$
\left\{\begin{array}{l}
\omega_{n}^{2}=\omega_{k}^{2}, \text { when } n=k \\
\omega_{n}^{2}=\omega^{2}, \text { when } n \neq k
\end{array}\right.
$$

For this special case, the following equations holds

$$
\left\{\begin{array}{l}
\sum_{n=1}^{N} \cos ^{2} \phi_{n}=\frac{N}{2} \quad N \geq 3 \\
\sum_{n=1}^{N} \sin ^{2} \phi_{n}=\frac{N}{2} \quad N \geq 3 \\
\sum_{n=1}^{N} \cos \phi_{n} \sin \phi_{n}=0
\end{array}\right.
$$

Applying (27) to (25), the SPEB without MT selection (i.e. $\mathcal{P}_{N}$ ) and SPEB with the $N-1$ selected MTs (i.e. $\mathcal{P}_{N-1}$ ) reads

$$
\begin{gathered}
\mathcal{P}_{N}=\frac{\frac{N-1}{\beta}+\frac{1}{\beta_{k}}}{\frac{N^{2}}{4 \beta^{2}}-\frac{N}{2 \beta^{2}}+\frac{N}{2 \beta \beta_{k}}} \\
\mathcal{P}_{N-1}=\frac{4 \beta(N-1)}{N^{2}-2 N}
\end{gathered}
$$

where $\beta=\omega^{2}+\varepsilon^{2} d^{2}, \beta_{k}=\omega_{k}^{2}+\varepsilon^{2} d^{2}$. It is found that

$$
\mathcal{P}_{N}<\mathcal{P}_{N-1}
$$

and

$$
\lim _{\omega_{k}^{2} \rightarrow \infty} \mathcal{P}_{N}=\mathcal{P}_{N-1}
$$

(30) indicate that with MT selection, the training overhead decreases by 1 with the paid of accuracy. (31) indicate that there is no accuracy degradation when $\omega_{k}^{2}$ is infinity. Let the accuracy degradation satisfying the following equation

$$
\mathcal{P}_{N} \geq \eta \mathcal{P}_{N-1}
$$

where $\eta \in[0,1)$. Then, (32) can be rewritten as

$$
\frac{\beta_{k}}{\beta} \geq \rho(N) \triangleq \frac{2 \eta(N-1)-(N-2)}{(N-1)(N-2)(1-\eta)}
$$

If $\beta_{k}$ satisfying (33), the $k$-th MT can be excluded with required accuracy degradation. $\eta$ can be set large enough for 
TABLE I

MT SELECTION SCHEME
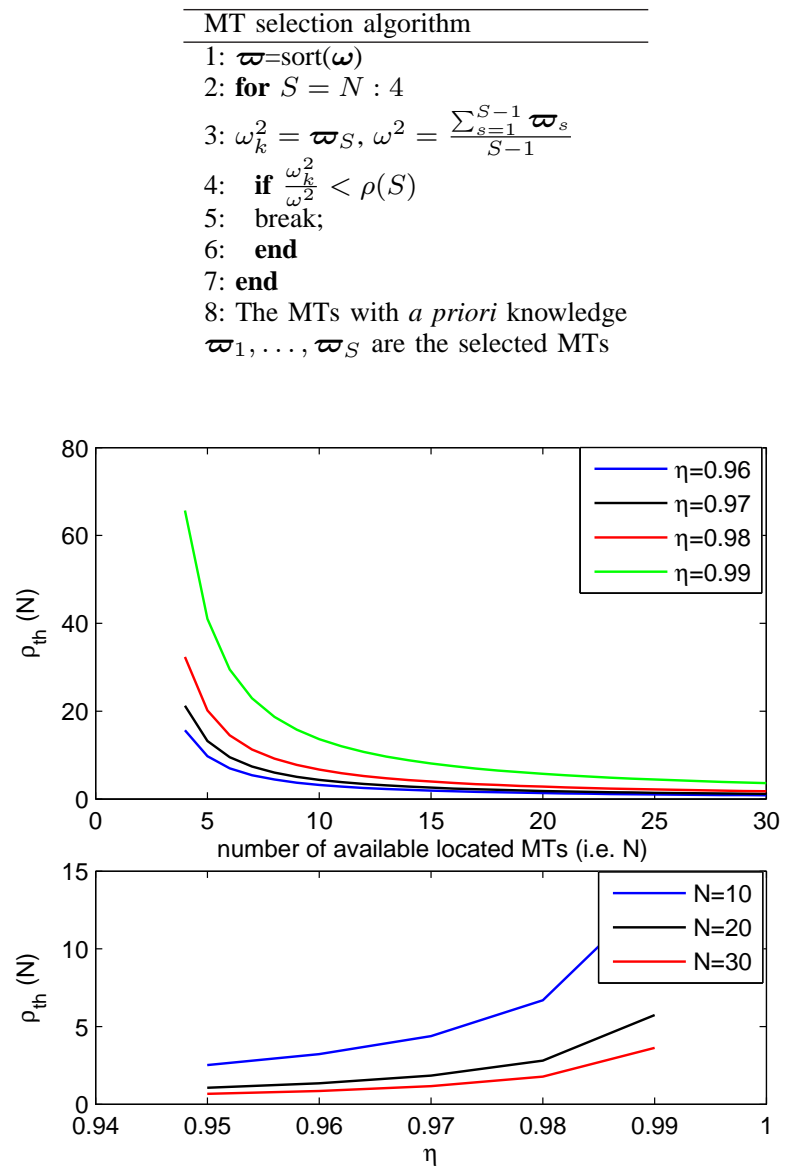

Fig. 2. $\rho(N)$ as a function of $\eta$ and $N$

an acceptable accuracy degradation. At this stage, the problem we want to address is how many and which located MTs need to be selected for a general case. Since the variance of the distance estimation is not known a priori, we simply set $\varepsilon^{2} d^{2}=0$, then (33) can be further written as

$$
\frac{\omega_{k}^{2}}{\omega^{2}} \geq \rho(N)
$$

$\omega^{2} \rho(N)$ is the minimum requirement of $\omega_{k}^{2}$ in order to exclude the $k$-th MT. According to the requirement in (34), the proposed MT selection scheme is presented in Tab. I. sort(·) denotes the MATLAB function, which sorts the elements of vector in ascending order. After MT selection using the algorithm in Tab. I, the training overhead is decreased by $N-S$. Since the proposed scheme is obtained from a special case, the accuracy degradation no longer satisfy (32). The accuracy degradation is evaluated using simulations in Section IV.

\section{Simulation Results And Discussion}

As shown in Tab. I, the proposed MT selection scheme depend on the threshold $\rho(N)$, which is related to $N$ and $\eta$.
TABLE II

PARAMETERS FOR SIMULATIONS

\begin{tabular}{lr} 
Parameters & Value \\
\hline$\omega$ & $5(\mathrm{~m})$ \\
$\sigma$ & $6(\mathrm{~dB})$ \\
$\gamma$ & 4 \\
$\mathrm{R}$ & $100(\mathrm{~m})$
\end{tabular}

The numerical results in Fig. 2 shows $\rho(N)$ as a function of $\eta$ and $N$. It is observed from the upper plot that $\rho(N)$ decreases with $N$ increases. This means that with fixed $\omega$, a larger $N$ leads to a smaller minimum requirement of $\omega_{k}^{2}$ in order to exclude the $k$-th located MT. It is observed from the lower plot that $\rho(N)$ increases with $\eta$ increases. This means that with fixed $\omega$, a larger $\eta$ leads to a larger minimum requirement of $\omega_{k}^{2}$ in order to exclude the $k$-th located MT.

The simulation results in Fig. 3 shows the effect of $N$ and $\eta$ on SPEB and training overhead. Fig. 4 shows the effect of $\eta$ on SPEB and training overhead. The parameters for simulations are shown in Fig. 3 and Fig. 4. For each realization, $N$ located MTs and one un-located MT are generated uniformly within the circle with radius $R$, as shown in Fig. 1. Assuming the elements in $\boldsymbol{\omega}$ subject to independent Rayleigh distribution. The variance of the Rayleigh random variables is $\frac{(4-\pi) 10^{2}}{2}$ $\left(\mathrm{m}^{2}\right)$. For each realization, the SPEB are calculated for the scheme without MT selection, with proposed MT selection, and with random MT selection, respectively. For the scheme without MT selection, all the $N$ located MTs are used to calculate the SPEB. For the proposed MT selection scheme, $S$ located MTs are selected according to the algorithm in Tab. I. Then, the SPEB is calculated using (25). For random MT selection scheme, $S$ located MTs are selected randomly from the $N$ MTs to calculate the SPEB. Thus, the training overhead is the same for the proposed scheme and random selection scheme. For performance comparison, the SPEB and training overhead is averaged over 10000 realizations.

The SPEB curve without selection in Fig. 3 shows that under the condition of imperfect location knowledge, the accuracy increases with the increases of the number of involved located MTs. It is observed from the upper and lower plot of Fig. 3 that, compared with the scheme without MT selection, the proposed MT selection scheme can reduce training overhead with the paid of accuracy in terms of SPEB. And the proposed MT selection offer better accuracy than random MT selection with the same training overhead.

In addition, Fig. 3 shows that for larger $N$, the proposed scheme can reduce more training overhead. This is because for larger $N$, the minimum requirement of $\omega_{k}^{2}$ is smaller, as discussed previously. Thus, more located MTs satisfy the requirement in (33). Fig. 4 shows that for larger $\eta$, the proposed scheme reduce less training overhead. This is because for larger $\eta$, the minimum requirement of $\omega_{k}^{2}$ is larger. Thus, less located MTs satisfy the requirement in (33). 


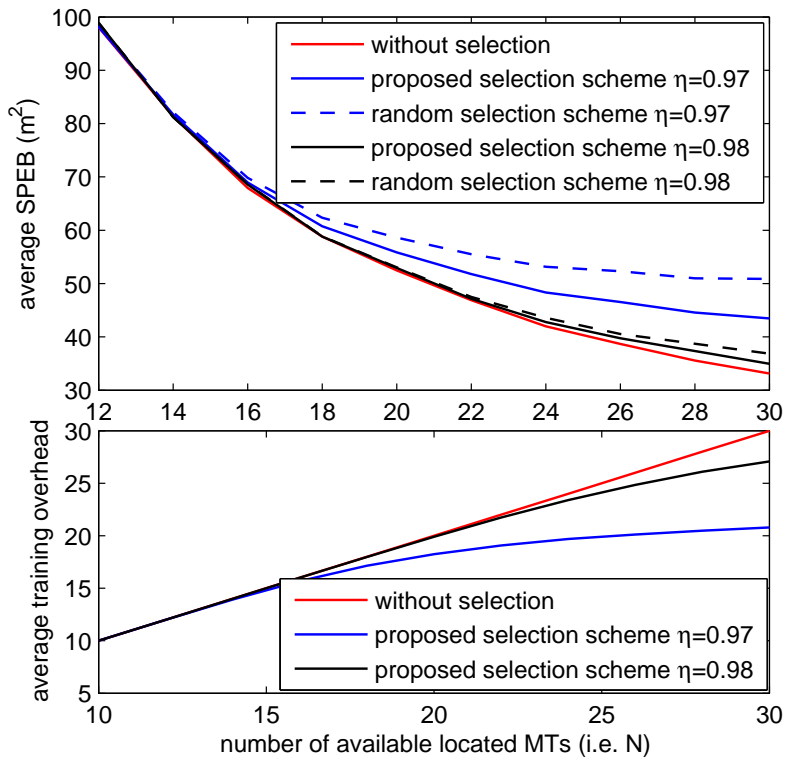

Fig. 3. average SPEB and average training overhead as a function of $N$

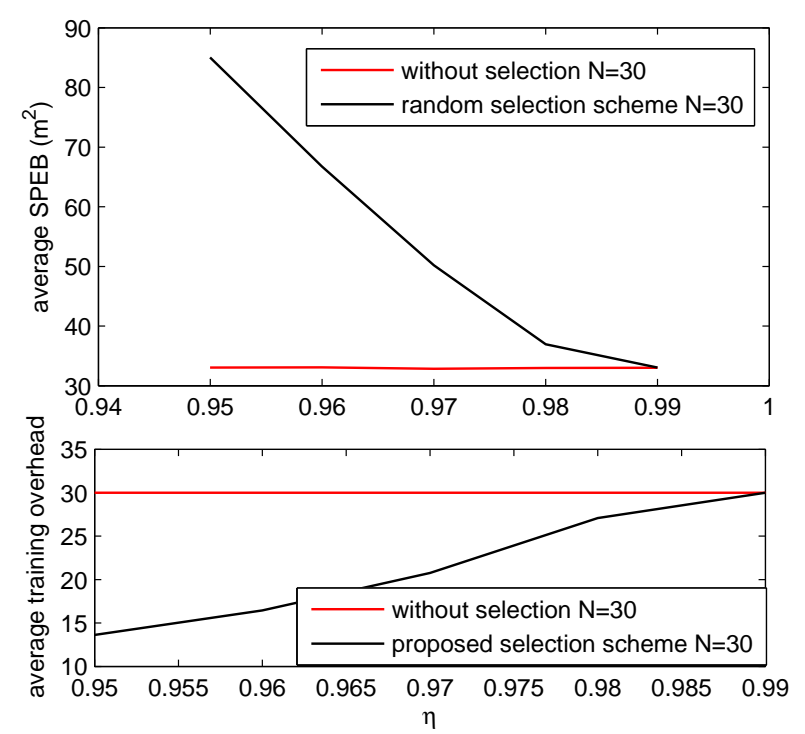

Fig. 4. average SPEB and average training overhead as a function of $\eta$

\section{CONCLUSIONS}

This paper considered cooperative localization in cellular networks. In this scenario, several located MTs are employed to find the location of an un-located MT. The located MTs sent training sequences in the uplink, then the un-located MT perform distance estimation using received signal strength techniques. The localization accuracy of the un-located MT is characterized in terms of SPEB. By taking into account imperfect a priori location knowledge of the located MTs, the SPEB was derived in a closed-form. The closed-form indicated that the effect of the imperfect knowledge on SPEB is equivalent to the increase of the variance of RSS-based distance estimation. Moreover, based on the obtained closedform, a MT selection scheme was proposed to decrease the number of located MTs sending training sequences, thus reduce the training overhead for localization. The proposed scheme can adaptively select the located MTs according to the a priori knowledge and the number of located MTs. The simulation results shown that the proposed scheme can reduce the training overhead with the paid of accuracy. And with the same training overhead, the accuracy of the proposed scheme is better than that of random selection.

\section{ACKNOWLEDGEMENT}

This work has been performed in the framework of the ICT project ICT- 248894 WHERE 2, which is partly funded by the European Union FP7.

\section{REFERENCES}

[1] Y. Shen and M. Z. Win, "Fundamental limits of wideband localization - part I: A general framework", IEEE Trans. Inf. Theory, vol. 56, no. 10, pp. 4956-4980, Oct. 2010.

[2] J. Caffery and G. L. Stuber, "Subscriber location in CDMA cellular networks", IEEE Trans. Veh. Technol, vol. 47, no. 2, pp. 406-416, May. 1998.

[3] Y. Zhao, "Standardization of mobile phone positioning for 3G systems", IEEE Commun. Mag., vol. 40, pp. 108-116, Jul. 2002.

[4] A. J. Weiss, "On the accuracy of a cellular location system based on RSS measurements", IEEE Trans. Veh. Technol, vol. 52, no. 6, pp. 1508-1518, Nov. 2003.

[5] C. Mensing, S. Sand, A. Dammann, and W. Utschick, "Data-Aided Location Estimation in Cellular OFDM Communications Systems", in Proc. IEEE Global Telecommun. Conf., December 2009.

[6] 3GPP TR 45.811, "Feasibility study on uplink TDOA in GSM and GPRS", http://www.3gpp.org, Jun. 2002

[7] 3GPP TS 36.305, "Stage 2 functional specifications of UE positioning in E-UTRAN", http://www.3gpp.org, May. 2009.

[8] A. H. Sayed, A. Tarighat, and N. Khajehnouri, "Network-based wireless location”, IEEE Signal Process. Mag., vol. 22, no. 4, pp. 12-23, Jul. 2005.

[9] N. Patwari, J. N. Ash, S. Kyperountas, and A. O. Hero III, "Locating the nodes: cooperative localization in wireless sensor networks", IEEE Signal Process. Mag., vol. 22, no. 4, pp. 54-69, Jul. 2005.

[10] G. Mao, B. Fidan, and B. D. O. Anderson, "Wireless sensor network localization techniques", Comput. Netw., vol. 51, no. 10, pp. 2529-2553, Jan. 2007.

[11] S. Frattasi, M. Monti, and R. Prasad, "Cooperative mobile user location for next-generation wireless cellular networks", in Proc. IEEE Int. Conf. Commun., Jun. 2006.

[12] F. L. Piccolo, "A new cooperative localization method for UMTS cellular networks", in Proc. IEEE Global Telecommun. Conf., Dec. 2008.

[13] Y. Zhang, Q. Cui, and X. Tao, "Cooperative group localization for 4G wireless networks", in Proc. IEEE Veh. Technol. Conf., Apr. 2009.

[14] Y. Qi, H. Kobayashi, and H. Suda, "Analysis of wireless geolocation in a non-line-of-sight environment", IEEE Trans. Wireless Commun., vol. 5, no. 3, pp. 672-681, Mar. 2006.

[15] A. Catovic and Z. Sahinoglu, "The cramer-rao bounds of hybird TOA/RSS and TDOA/RSS location estimation schemes", IEEE Commun. Lett., vol. 8, no. 10, pp. 626-628, Oct. 2004.

[16] H. L. Van Trees, Dectection, Estimation, and Modulation Theory-Part I, Hoboken, NJ: Wiley, 1968.

[17] R. W. Ouyang, A. K. S. Wong, and C. T. Lea, "Received signal strengthbased wireless localization via semidefine programming: noncooperative and cooperative schemes", IEEE Trans. Veh. Technol, vol. 59, no. 3, pp. 1307-1318, Mar. 2010.

[18] Y. Qi and H. Kobayashi, "On relation among time delay and signal strength based geolocation methods", in Proc. IEEE Global Telecommun. Conf., December 2003. 